\title{
Selective Roles for Hippocampal, Prefrontal Cortical, and Ventral Striatal Circuits in Radial-Arm Maze Tasks With or Without a Delay
}

\author{
Stan B. Floresco, Jeremy K. Seamans, and Anthony G. Phillips \\ Department of Psychology, University of British Columbia, Vancouver, British Columbia, Canada V6T $1 Z 4$
}

The hippocampus, the prefrontal cortex, and the ventral striatum form interconnected neural circuits that may underlie aspects of spatial cognition and memory. In the present series of experiments, we investigated functional interactions between these areas in rats during the performance of delayed and nondelayed spatially cued radial-arm maze tasks. The twophase delayed task consisted of a training phase that provided rats with information about where food would be located on the maze 30 min later during a test phase. The single-phase nondelayed task was identical to the test phase of the delayed task, but in the absence of a training phase rats lacked previous knowledge of the location of food on the maze. Transient inactivation of the ventral CA1/subiculum (vSub) by a bilateral injection of lidocaine disrupted performance on both tasks. Lidocaine injections into the vSub on one side of the brain and the prefrontal cortex on the other transiently disconnected these two brain regions and significantly impaired foraging during the delayed task but not the nondelayed task. Transient disconnections between the vSub and the nucleus accumbens produced the opposite effect, disrupting foraging during the nondelayed task but not during the delayed task. These data suggest that serial transmission of information between the vSub and the prefrontal cortex is required when trial-unique, short-term memory is used to guide prospective search behavior. In contrast, exploratory goal-directed locomotion in a novel situation not requiring previously acquired information about the location of food is dependent on serial transmission between the hippocampus and the nucleus accumbens. These results indicate that different aspects of spatially mediated behavior are subserved by separate, distributed limbiccortical-striatal networks.

Key words: ventral CA1/subiculum; prelimbic cortex; nucleus accumbens; spatial memory; neural networks; lidocaineinduced reversible lesions; rats
The ability to locate and retrieve food efficiently is an essential survival strategy for rodents and other mammals, and the neural substrates of foraging behavior have been studied extensively using radial-arm maze procedures pioneered by Olton and Samuelson (1976). Much of this work has focused on the role of the hippocampus in mediating foraging behavior using spatial cues (Olton and Papas, 1979; Jarrard, 1993). However, recent data have emphasized a role for other neural structures, such as the prelimbic (PL) region of the rat prefrontal cortex (PFC) and nucleus accumbens (N.Acc.) in spatially based foraging behaviors on a radial maze (Seamans and Phillips, 1994; Seamans et al., 1995). The ventral CA1/subiculum (vSub) region of the hippocampus projects to both the PL (Jay and Witter, 1991; Conde et al., 1995) and the medial N.Acc. (Groenewegen et al., 1987; Brog et al., 1993). In addition, the PL sends dense projections to the N.Acc. (Groenewegen et al., 1987; Sesack et al., 1989). Using these pathways, spatial information essential for foraging behavior could be relayed from the hippocampus to the N.Acc. and/or PFC to guide foraging behavior according to the demands of specific radial-arm maze tasks.

The PL appears to play a selective role in a delayed choice but

Received Oct. 10, 1996; revised Dec. 17, 1996; accepted Dec. 18, 1996.

This research was supported by a grant from the Natural Sciences and Engineering Research Council (NSERC) of Canada to A.G.P. S.B.F. is a recipient of an NSERC scholarship, and J.K.S. holds a University of British Columbia Graduate Fellowship. We thank Ms. Penny Lam and Mr. Tony Drew for their assistance with behavioral testing, Dr. Charles D. Blaha and Mr. Jason Carr for helpful discussions, and Ms. Susanna K. Kovacs for editorial comments.

Correspondence should be addressed to Anthony G. Phillips, Department of Psychology, University of British Columbia, 2136 West Mall, Vancouver, British Columbia, Canada V6T 1 Z4.

Copyright (C) 1997 Society for Neuroscience $\quad 0270-6474 / 97 / 171880-11 \$ 05.00 / 0$ not a nondelayed version of the radial-arm maze task (Seamans et al., 1995). Delayed tasks such as this one (see Fig. $1 A$ ) bias rats to forage prospectively (Cook et al., 1985; Kesner, 1989), suggesting that the PL is involved in the planning of motor response strategies to ensure that food is located efficiently. Impairments on delayed spatial tasks after lesions to either the PFC (Kesner, 1989; Dunnett, 1990; Seamans et al., 1995) or the hippocampal formation (Kesner and DiMattia, 1987; Dunnett, 1990) suggest that these two brain regions may interact when an animal is foraging under these conditions.

Unlike the PL, the N.Acc. is involved in both delayed spatial win-shift (SWSh) and nondelayed random foraging (RF; see Fig. $1 B$ ) versions of radial-arm maze foraging tasks (Seamans and Phillips, 1994), indicating a general role for the N.Acc. in goaldirected locomotion (Kelley and Stinus, 1985; Mogenson et al., 1993). In nondelayed radial-arm maze tasks, both place- and movement-consistent firing have been recorded from neurons in the N.Acc. (Lavioe and Mizumori, 1994), resembling similar activity observed from vSub neurons (Barnes et al., 1990; Jung et al., 1994; Poucet et al., 1994). Moreover, lesions of either the N.Acc. or the vSub disrupt performance on single-trial foraging tasks (Jarrard, 1993; Seamans and Phillips, 1994). These findings are consistent with an important role for a vSub-N.Acc. circuit in situations in which the rat must navigate in a spatial environment without the benefit of previous knowledge of the probable location of food. This process is distinct from those using a prospective strategy to guide foraging that may be mediated by separate neural circuits involving the PFC-vSub or PFC-N.Acc.

The present study was designed to test the hypotheses that (1) foraging in a nondelayed condition is dependent on a direct 


\section{A The Delayed Spatial Win-Shift (SWSh) Paradigm}

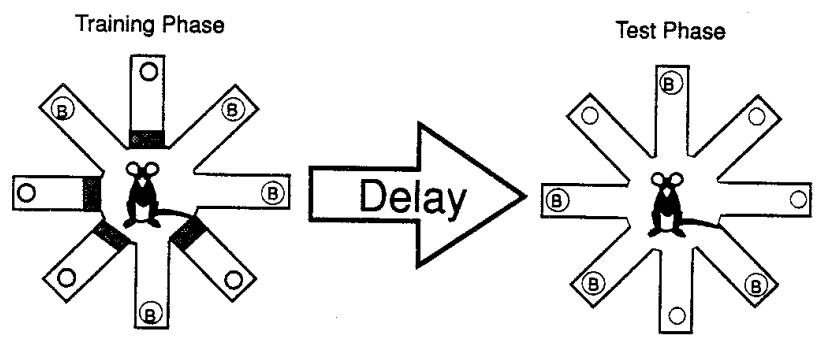

(B) Baited Arm

Blocked Arm

\section{B The Non-Delayed Random Foraging (RF) Paradigm}

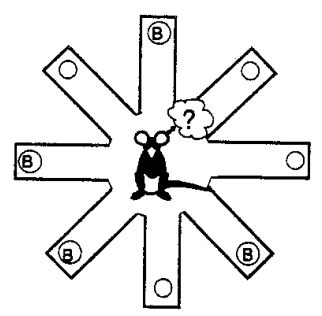

(B) Baited Arm

Figure 1. Diagrams of the delayed spatial win-shift (SWSh) and the random foraging (RF) eight-arm radial-maze tasks. $A$, The delayed SWSh task consists of a training and a test phase. During the training phase, 4 of 8 arms on a radial maze are randomly blocked, and the 4 remaining open arms are baited. Once the animal has retrieved the 4 pieces of food from the open arms, it is removed from the maze for a delay (ranging from 5 to $30 \mathrm{~min}$ ). After the delay, the animal is placed back onto the maze for the test phase. The arms that were blocked previously are now open and baited. The rat must remember which arms were previously blocked and enter them to receive the food reward. $B$, The nondelayed RF task consists of one phase. Four arms are randomly baited each day. The optimal foraging strategy entails entering the arms in a nonrepetitive manner. Unlike the test phase of the delayed SWSh task, the animal has no previous knowledge of the location of food at the beginning of a nondelayed RF trial.

interaction between the vSub and N.Acc., whereas (2) foraging guided by trial unique information stored in memory during a delay is mediated by a transcortical network connecting the hippocampus and the PL region of the PFC (Doyère et al., 1991; Jay and Witter, 1991; Conde et al., 1995). An asymmetric disconnection procedure was used to block the transmission of information within specific pathways in each hemisphere. Specifically, a unilateral lidocaine-induced lesion of the origin of parallel efferent pathways in one hemisphere was used in combination with a contralateral lidocaine-induced lesion in one of the target areas of the efferent projection in the other hemisphere. This procedure has proven to be particularly useful in defining the route of serial information transfer between different brain regions in both rats (Everitt et al., 1991) and primates (Gaffan and Harris, 1987; Gaffan et al., 1988, 1989). Two patterns of bilateral inactivation were used in the following experiments: unilateral reversible le- sions of the vSub in combination with a contralateral lesion of either (1) the PL region of the PFC or (2) the N.Acc. Bilateral inactivations occurred before the test phase of the delayed SWSh task or before the nondelayed RF task.

Previous research in our laboratory has investigated the effects of bilateral inactivation of the PL (Seamans et al., 1995) and the N.Acc. (Seamans and Phillips, 1994) but not the vSub. The asymmetric disconnection procedure is based on the assumption that bilateral lesions of each of the target areas will produce a specific behavioral deficit. Thus, an initial experiment assessed the effect of bilateral reversible lesions of the vSub before the training or test phase of the delayed SWSh or before the RF task. This experiment, in combination with the disconnection experiments described above, provides a detailed analysis of the role of these cortico-limbic networks in spatially guided choice behavior in the radial-arm maze.

Some of these findings were reported in abstract form at the Annual Meeting of the Society for Neuroscience, San Diego (1995).

\section{MATERIALS AND METHODS}

\section{Subjects}

The subjects were male Long-Evans rats weighing between 300 and 450 $\mathrm{gm}$ before surgery. All rats were given free access to water and were maintained at $85 \%$ of their free-feeding weight by providing $25-30 \mathrm{gm}$ of Purina lab chow pellets once daily. Rats were tested 5-7 d per week.

\section{Surgery}

Rats were anesthetized with $100 \mathrm{mg} / \mathrm{kg}$ ketamine hydrochloride and 7 $\mathrm{mg} / \mathrm{kg}$ xylazine. Twenty-three gauge stainless-steel guide cannulae were implanted into the brain regions of interest using standard stereotaxic techniques. The stereotaxic coordinates (flat skull) were derived from Paxinos and Watson (1986). For the bilateral vSub inactivation experiments, one set of cannulae were implanted bilaterally into the vSub region of the hippocampus: AP, $-6.0 \mathrm{~mm}$ from bregma; $\mathrm{ML}, \pm 5.5 \mathrm{~mm}$ from midline; DV, $-5.3 \pm 0.3 \mathrm{~mm}$ from dura. Rats in the disconnection experiments were implanted with two sets of bilateral cannulae, one pair into the vSub and a second pair into either the PL region of the medial PFC (AP, $+2.6 \mathrm{~mm}, \mathrm{ML}, \pm 0.7 \mathrm{~mm}$ from bregma; DV, $-3.0 \mathrm{~mm}$ from dura) or the medial shell region of the N.Acc. (AP, $+1.6 \mathrm{~mm}$ from bregma, $\pm 1.3 \mathrm{~mm}$ from midline, $-6.0 \mathrm{~mm}$ from dura). Thirty-gauge obdurators flush with the end of the guide cannulae remained in place until the injections were made. Each rat was given at least $7 \mathrm{~d}$ to recover from surgery before testing.

\section{Microinfusion procedure}

On injection days, the obdurators were removed and 30-gauge stainlesssteel injection cannulae were inserted $0.8 \mathrm{~mm}$ beyond the tip of the guide cannulae. Lidocaine $(20 \mu \mathrm{g}$ in $0.5 \mu \mathrm{l}$ of saline, Astra Pharmaceuticals and Research Biochemicals) or vehicle injections were delivered at a rate of $0.5 \mu \mathrm{l} / 1.2 \mathrm{~min}$ by a microsyringe pump (Sage Instruments, Model 341). Injection cannulae were left in place for an additional $1 \mathrm{~min}$ after each injection to allow for diffusion. Each rat remained in its home cage for an additional 3 min before being placed on the maze.

There are different estimates of the functional spread of lidocaine within the brain that appear to depend on the concentration and rate of infusion. Using an infusion rate of $1 \mu \mathrm{l} / \mathrm{min}$, Welsh and Harvey (1991) estimated that the functional spread of $20 \mu \mathrm{g}$ of lidocaine in $1 \mu \mathrm{l}$ of saline is $1.4 \mathrm{~mm}$ in the cerebellum from the site of infusion. The functional spread of the same concentration of lidocaine in the occulomotor nucleus was estimated to be $0.5 \mathrm{~mm}$ with an infusion rate of $4 \mu \mathrm{l} / 15 \mathrm{~min}$ (Albert and Madryga, 1980). Furthermore, infusions of $20 \mu \mathrm{g}$ of lidocaine in $1 \mu \mathrm{l}$ of saline at a rate of $1 \mu \mathrm{l} / 2 \mathrm{~min}$ into either the PL or the anterior cingulate cortex of the rat, which are separated by $1.5 \mathrm{~mm}$, produced distinctly different patterns of impairment on a battery of radial-arm maze tasks (Seamans et al., 1995). These results are consistent with an effective functional spread of lidocaine being no greater than $1.5 \mathrm{~mm}$. Because half the volume $(0.5 \mathrm{ul})$ was used the present study, a functional spread of no greater than $1 \mathrm{~mm}$ would be expected. 


\section{Apparatus}

An eight-arm radial maze was used for all experiments. The maze had an octagonal center platform $40 \mathrm{~cm}$ in diameter connected to eight, equally spaced arms, each measuring $50 \mathrm{~cm} \times 9 \mathrm{~cm}$, with a cylindrical food cup at the end (see Fig. 1). Removable pieces of white opaque plastic $(9 \mathrm{~cm} \times 13 \mathrm{~cm})$ were used to block the arms of the maze. The maze was elevated $40 \mathrm{~cm}$ from the floor and was surrounded by numerous extra maze cues (i.e., cupboards, posters, doors, the experimenter, etc.) in a room $4 \mathrm{~m} \times 5 \mathrm{~m} \times 3 \mathrm{~m}$, which was illuminated with overhead fluorescent lights $(100 \mathrm{~W})$.

\section{Foraging tasks}

The two foraging tasks used in the present study were the delayed SWSh task and the nondelayed RF task.

Delayed SWSh task. This task was adapted from Packard et al. (1990) and has been described in detail elsewhere (Seamans and Phillips, 1994; Seamans et al., 1995) (see Fig. $1 A$ ). On the first $2 \mathrm{~d}$ of testing, rats were habituated to the maze environment. Subsequent training trials were given once daily. These trials consisted of a training phase and a test phase, separated by a delay. Before the training phase, a set of four arms was chosen randomly and blocked. Food pellets (Bioserv, Frenchtown, $\mathrm{NJ}$ ) were placed in the food cups of the four remaining open arms During the training phase, each rat was given 5 min to retrieve the pellets from the four open arms and then was returned to its home cage for the delay period (see below). During the test phase of each daily trial, all arms were open, but only the arms that were previously blocked contained food. Rats were allowed a maximum of 5 min to retrieve the four pellets during the test phase.

The initial delay between training and test phases was $5 \mathrm{~min}$. After achieving criterion performance in which all four pellets were retrieved in five or fewer choices during the test phase for two consecutive days, the delay was increased to $30 \mathrm{~min}$. The first intracranial injections were administered after attaining two consecutive days of criterion performance at a 30 min delay. After the first injection day, animals were again retrained to a criterion performance. The next day, a second intracranial injection was administered. This procedure was repeated until an animal had received all of the designated sequence of injections according to the protocols described below (see Design and Procedure).

On injection days, the number and order of arm entries were recorded. An arm entry was recorded when a rat moved down the entire length of an arm and reached the food cup at the end of the arm. Errors were scored as entries into nonbaited arms and further broken down into two error subtypes. An across-phase error was defined as any initial entry to an arm that had been entered previously during the training phase. A within-phase error was any reentry into an arm that had been entered earlier during the test phase. The latencies to reach the food cup of the first arm visited and to complete the phase were also recorded.

Nondelayed RF task. This task has also been described elsewhere (Seamans and Phillips, 1994; Seamans et al., 1995) (see Fig. 1B). Habituation to the maze during the first $2 \mathrm{~d}$ of training was identical to the delayed SWSh procedure described above. On subsequent daily trials, animals were required to forage for pellets placed at random in the food cups of 4 of the 8 arms. A novel set of arms was baited each day. Animals were trained to a criterion of no more than one reentry error per daily trial for four consecutive days. The day after criterion performance was achieved, the first intracranial injections were administered. After the first injection day, animals were retrained to criterion for two consecutive days. As with the delayed SWSh task, this procedure was repeated until each animal had received all of the designated injections.

Errors were scored as reentries into arms entered previously within a trial. These errors were broken down further into reentries into baited arms (arms that had been baited at the start of the trial) and reentries to nonbaited arms (arms that were not baited before the start of the trial). The number of reentries errors made on each of the injection days was recorded and used for data analysis. As with the delayed SWSh paradigm, the latencies to reach the first food cup (either baited or nonbaited) after being placed on the maze and the time required to retrieve all four pellets were also recorded.

\section{Design and procedure}

Experiment 1: reversible, bilateral vSub lesions. A within-subjects design was used for all three parts of Experiment 1. Three groups of rats with bilateral cannulae implanted into the vSub were trained on either the delayed SWSh task or the RF task. On the first injection days, three separate groups of rats received bilateral infusions of either lidocaine or saline into the vSub before the nondelayed RF task (group 1), before the training phase (group 2), or before the test phase of the delayed SWSh (group 3). Animals were subsequently retrained until they re-attained criterion performance. On the day after criterion was re-attained, a second infusion of either saline or lidocaine was administered in a counterbalanced order.

Experiment 2: disconnection lesions between the $v$ Sub and the PL or the N.Acc. A within-subjects design was used for all four parts of Experiment 2. Two groups of rats were implanted bilaterally with two sets of cannulae into both the vSub and the PL. After recovery from surgery, they were trained on either the delayed SWSh task or the nondelayed RF task. Two other groups of animals with cannulae implanted bilaterally into the vSub and the N.Acc. also were trained on either task.

Animals in each of the disconnection lesion experiments received a total of four injection days, either before the test phase of the delayed SWSh task or before the nondelayed RF task. The following combinations of asymmetrical bilateral inactivations were used: (1) a unilateral inactivation of the vSub in combination with a contralateral inactivation of either the PL or the N.Acc. (disconnection); (2) a unilateral inactivation of the vSub in combination with a saline injection into the contralateral PL or N.Acc.; (3) a unilateral inactivation of the PL or N.Acc. in combination with a saline injection into the contralateral vSub; and (4) unilateral injections of saline into the vSub and saline injections into either the contralateral PL or the N.Acc. The order of injections was counterbalanced between animals using a quasi-Latin square design. The counterbalancing was designed to ensure that a given sequence of injections was not repeated. The hemisphere (left or right) used for the first injection was also counterbalanced and was alternated for subsequent injections. Each animal was tested separately on the same foraging task, and the injection procedure was repeated until the animal had been tested four times with each sequence of intracranial injections.

\section{Histology}

After completion of behavioral testing, the rats were killed in a carbon dioxide chamber. Brains were removed and fixed in a $10 \%$ formalin solution. The brains were frozen and sliced in $50 \mu \mathrm{m}$ sections before being mounted and stained with cresyl violet. Placements were verified with reference to the neuroanatomical findings of Jay and Witter (1991), Brog et al. (1992), Conde et al. (1995), Groenewegen et al. (1987), and Paxinos and Watson (1986).

\section{Data analysis}

The number and type of errors made on the day before the first injection sequence and for all injection days for each experiment were analyzed using separate three-way, between/within, mixed design ANOVAs with the Injection order (lidocaine or saline first) as a between-subjects factor, and Treatment day and Error type as two within-subjects factors. Main effects of treatment were analyzed further using Tukey's post hoc tests for repeated measures. Whenever a significant main effect of treatment was observed, one planned comparison was made, analyzing the number of each type of error made on lidocaine injection days.

The latency data to reach the first food cup and the average time per subsequent choice on injection days were analyzed with a one-way repeated-measures ANOVA. The average time per subsequent choice was calculated using the following formula: [(Time to complete trial Time to initiate trial)/(Number of choices for trial)].

Whenever a significant main effect of treatment was observed in Experiment 2, three one-way ANOVAs were conducted, assessing the number of errors made on tests after a unilateral inactivation of the vSub, PL, or N.Acc., as well as after disconnection inactivations, with the side of the injection as a between-subjects factor. This analysis was conducted to rule out the possibility that unilateral inactivations in one hemisphere would lead to a greater increase in errors than inactivations of the other hemisphere.

\section{RESULTS}

\section{Experiment 1: reversible, bilateral vSub lesions Histology}

The location of the cannulae tips for all animals tested in Experiment 1 are shown in Figure 2. Some placements encroached slightly on the CA3/CA1-dentate gyrus border, but the behavior of these animals did not differ from those in which placements were located within the vSub region. 


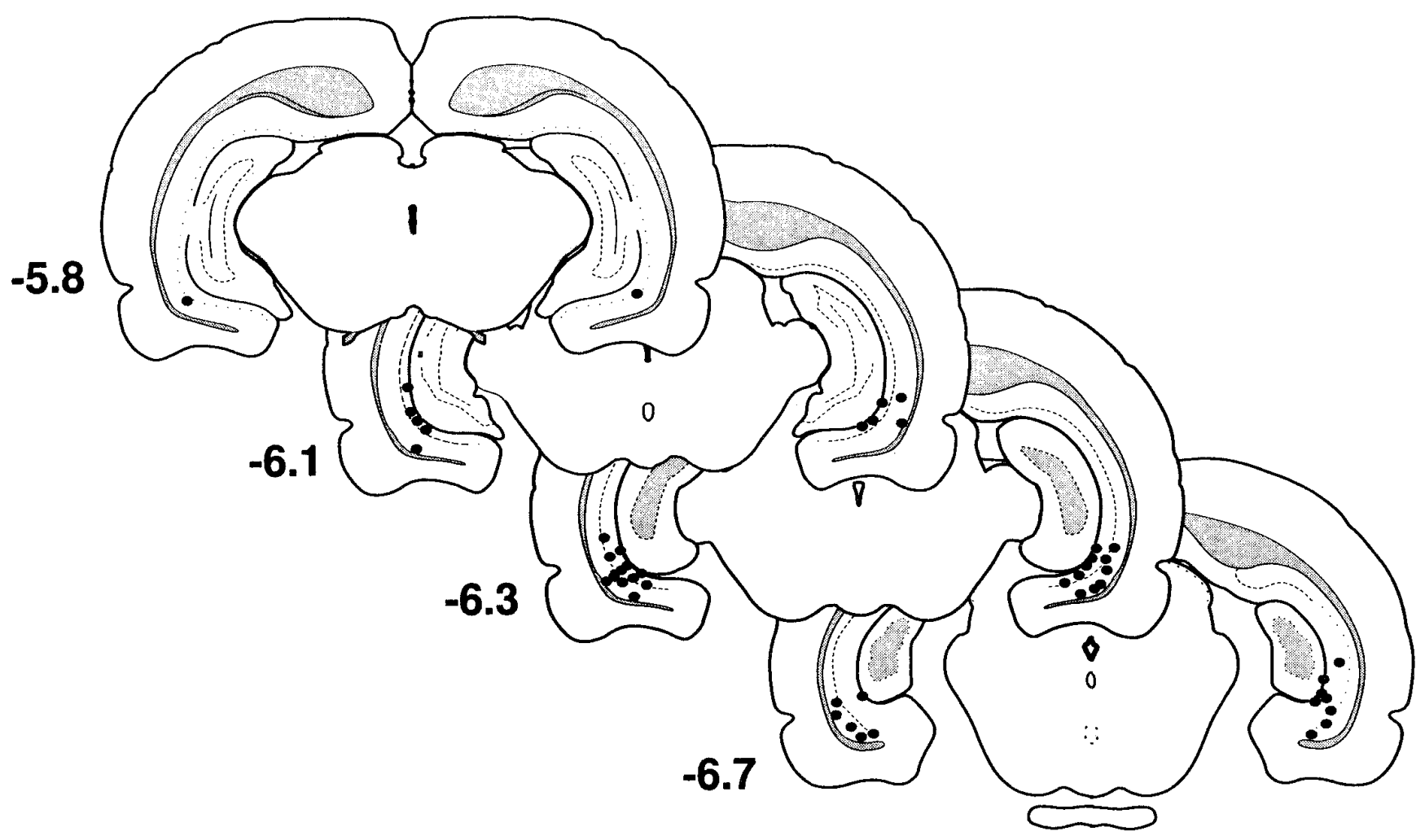

Figure 2. Histology from the bilateral vSub inactivation experiments. Location of cannulae tips (black circles) for all rats used for data analysis in Experiment 1. Plates are computer-generated adaptations from Swanson (1992) that were modified to resemble those from Paxinos and Watson (1986). Numbers beside each slide correspond to millimeters from bregma.

\section{Nondelayed $R F$}

Before a daily trial of the nondelayed RF task, eight rats received bilateral infusions of lidocaine or saline into the vSub, on separate days. The analysis of these data revealed a significant main effect of Treatment $\left(F_{(2,12)}=21.89, p<0.001\right)$. Tukey's post hoc analysis showed that rats made significantly more errors on lidocaine injection days relative to saline injection days or to the day before the first injection ( $p<0.001$; Fig. $3 A$ ). Subsequent analysis of the type of errors made on injection days revealed that animals receiving lidocaine infusions showed no perseverative tendencies and made the same number of revisits to both baited and nonbaited arms $\left(F_{(1,7)}=0.04\right.$, not significant; see Fig. $3 A$, inset $)$. There were no significant effects of Order of injection or Order $X$ Treatment interactions (all $F<1.2$, not significant). There also were no significant differences in the latencies to reach the first food cup or on the average time per subsequent choice for all three injection days (all $F<2.2$, not significant).

\section{Delayed SWSh, pretraining injections}

Before the training phase of the delayed SWSh task, eight animals received bilateral infusions of lidocaine or saline into the vSub on separate days. There were no significant differences in the number of errors made on either the training phase $\left(F_{(2,12)}=0.33\right.$, not significant; see Fig. $3 B$, inset $)$ or the test phase $\left(F_{(2,12)}=2.45\right.$, not significant; see Fig. $3 B$ ) on the day before the first injection and on saline or lidocaine injection days. There were also no significant effects of Injection Order or Order $\times$ Treatment interactions (all $F<2.0$, not significant). In addition, there were no significant differences in latencies to reach the first food cup or in the average time per subsequent choice of arms on both the test and training phases (all $F<1.3$, not significant).

\section{Delayed SWSh, pretest injections}

Before the test phase of the delayed SWSh task, nine rats received bilateral infusions of lidocaine or saline into the vSub on separate days. Analyses of the number of errors made on the day before the first injection and on saline and lidocaine injection days revealed a significant main effect of Treatment $\left(F_{(2,14)}=25.05, p<0.001\right.$; see Fig. 3C). Tukey's post hoc analysis for repeated measures showed that animals made significantly more errors on lidocaine injection days relative to both the day before the first injection and the saline injection days ( $p<0.001)$. Subsequent planned comparisons on the type of errors made on lidocaine injection days revealed that rats made significantly more across-phase errors than within-phase errors $(p<0.01$; see Fig. $3 C$, inset $)$. There were no significant effects of Injection Order or Treatment $\times$ Order interactions (all $F<1$, not significant). Likewise, there were no significant differences between injection days on the latencies to reach the first food cup or on the average time per subsequent choice (all $F<1.7$, not significant).

\section{Discussion}

Bilateral inactivation of the vSub disrupts performance on both the delayed and the nondelayed radial-arm maze tasks. This is consistent with a role for this region of the hippocampus in spatial memory. In a recent experiment, similar reversible lesions of the vSub in naive rats disrupted the acquisition of an escape response guided by spatial information in a Morris water maze (Floresco et al., 1996). This effect is attributed to a temporary blockade of access to information encoding the location of the escape platform, because rats were able to escape as efficiently as controls after the effect of lidocaine had dissipated. These data along with those of Poucet et al. (1991) support the theory that the ventral 
Non-delayed RF Injections

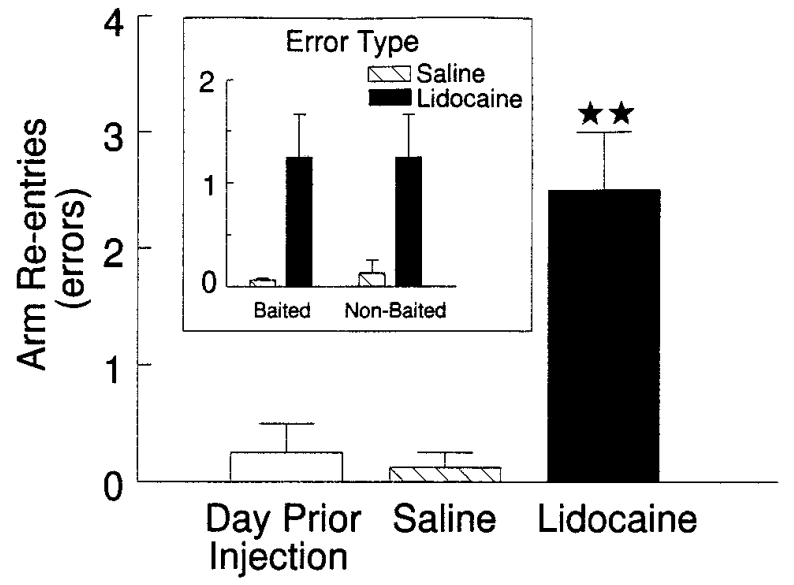

Delayed SWSh, Pretraining injections
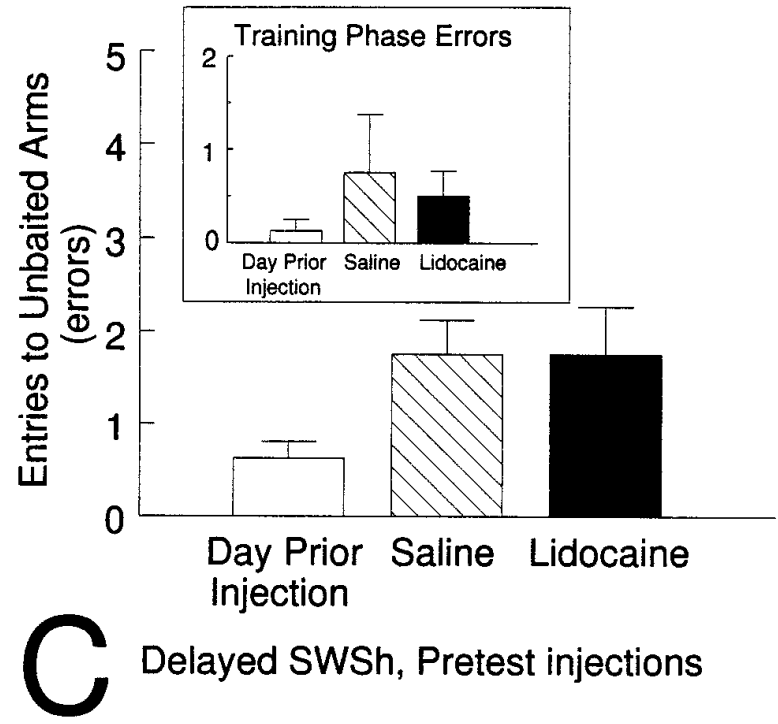

Delayed SWSh, Pretest injections

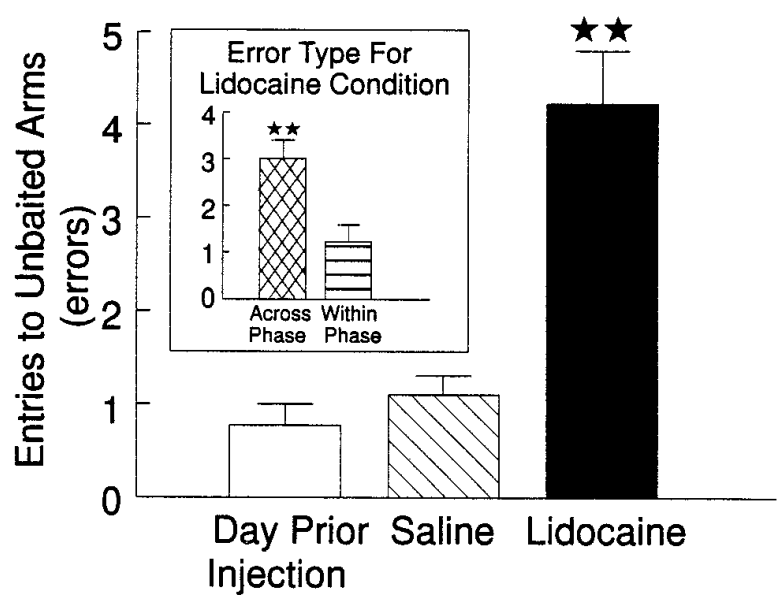

Figure 3. The effects of bilateral inactivation of the vSub on performance of delayed and nondelayed radial-arm maze tasks. $A$, Number of errors (mean \pm SEM) made by rats on the day before the first injection (open bar) and after infusions of saline (hatched bar) and lidocaine (black bar) hippocampus is involved in the short-term processing of spatial information, but not its storage over a delay. These data, in addition to the present set of experiments, are consistent with an extensive series of behavioral and electrophysiological studies implicating both the dorsal and ventral hippocampus in the formation of cognitive maps (O'Keefe and Nadel, 1978; Barnes et al., 1990; Morris et al., 1991; Jung et al., 1994; Sharp and Green, 1994). Disruption of spatially mediated foraging behavior by lidocaine injections into vSub, during both the delayed and the nondelayed tests, may be attributed to the blockade of transfer of spatial information, processed initially by the hippocampal formation, to forebrain structures required for subsequent processing and motor integration. This hypothesis was tested directly in Experiment 2 (see below).

In addition to the disruption of spatial information processing, hippocampal lesions also are associated with response perseveration (Devenport et al., 1981; Issacson, 1982; Packard et al., 1989). Superficially, the preponderance of "across-phase" errors during the test phase of the delayed SWSh task, after bilateral vSub inactivations, could be interpreted as a disruption of behavioral flexibility (i.e., predominately reentering arms that had been baited previously during the training phase). However, if behavioral inflexibility is a consistent effect of ventral hippocampal lesions, it follows that errors in the nondelayed RF task should consist primarily of reentries into previously baited arms. This pattern was not observed because baited and nonbaited arms were revisited with equal frequency in this latter task. Rather, the pattern of errors observed after pretest inactivation of the vSub in the delayed SWSh task may be better explained as a temporally graded spatial memory deficit (i.e., a greater disruption of memory for locations visited $30 \mathrm{~min}$ before the test phase vs arms visited more recently during the test phase). This interpretation is consistent with the finding that rats with fornix lesions demonstrated delay-dependent impairments on a delayed-matching-toposition paradigm (Dunnett, 1990).

\section{Experiment 2: disconnection lesions between the vSub and the PL or the N.Acc.}

\section{$P L-v$ Sub disconnections}

Nondelayed $R F$. Rats $(n=8)$ with two sets of bilateral cannulae implanted into the PL, and the vSub received the injection protocol described above before the nondelayed RF task on four separate days. Statistical analysis on the performance of these rats revealed no main effect of Treatment $\left(F_{(4,16)}=0.67\right.$, not significant; see Fig. $4 A$ ). Similarly, there were no main effects of Injection Order, Error type, or any significant interaction (all $F<1.2$,

$\leftarrow$

into the vSub before the nondelayed random foraging task. $* * p<0.001$ relative to saline and day previous. Inset shows number of revisits to baited arms and nonbaited arms on saline (hatched bar) and lidocaine (black bar) injection days. $B$, Number of errors (mean \pm SEM) made by rats during the test phase on the day before the first injection (open bar) and after infusions of saline (hatched bar) and lidocaine (black bar) into the vSub before the training phase of the delayed spatial win-shift task. Inset shows number of errors made during the training phase on the day before the first injection (open bar) and on saline (hatched bar) and lidocaine (black bar) injection days. $C$, Number of errors (mean \pm SEM) made by rats during the test phase on the day before the first injection (open bar) and after infusions of saline (hatched bar) and lidocaine (black bar) into the vSub before the test phase of the delayed spatial win-shift task. ${ }^{* *} p<$ 0.001 relative to saline and day previous. Inset shows number of acrossphase errors (cross-hatched bar) and within-phase errors (stripped bar) made by rats on lidocaine injection days. ${ }^{*} p<0.01$. 


\section{Non-delayed Random Foraging}
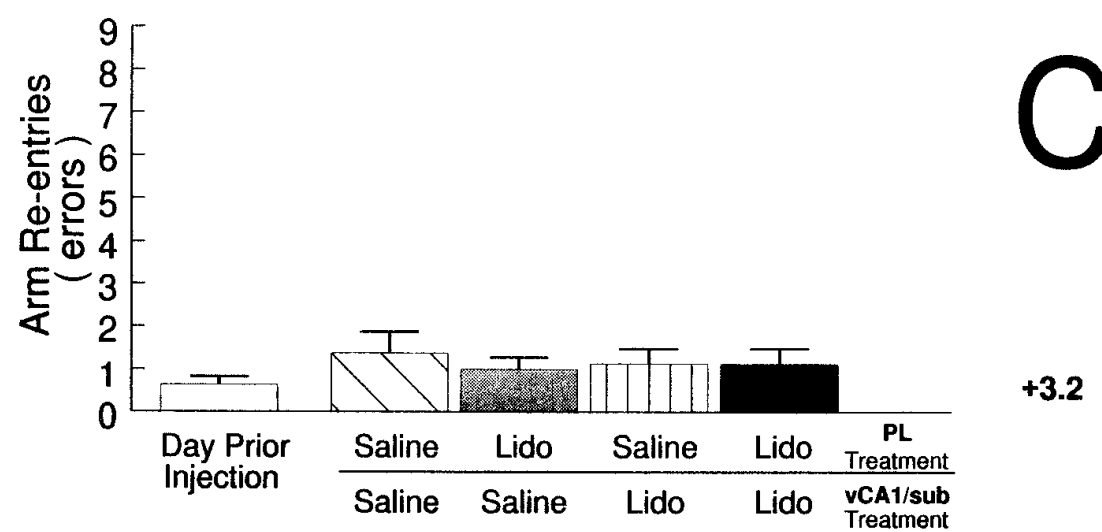

\section{Location of Cannulae Tips}

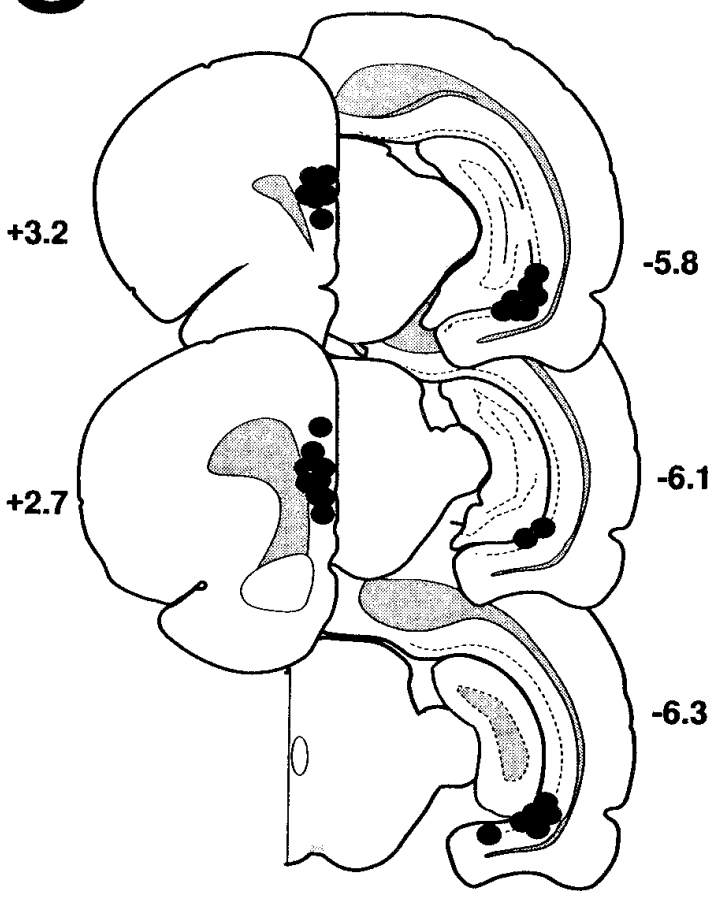

Delayed Spatial Win-Shift

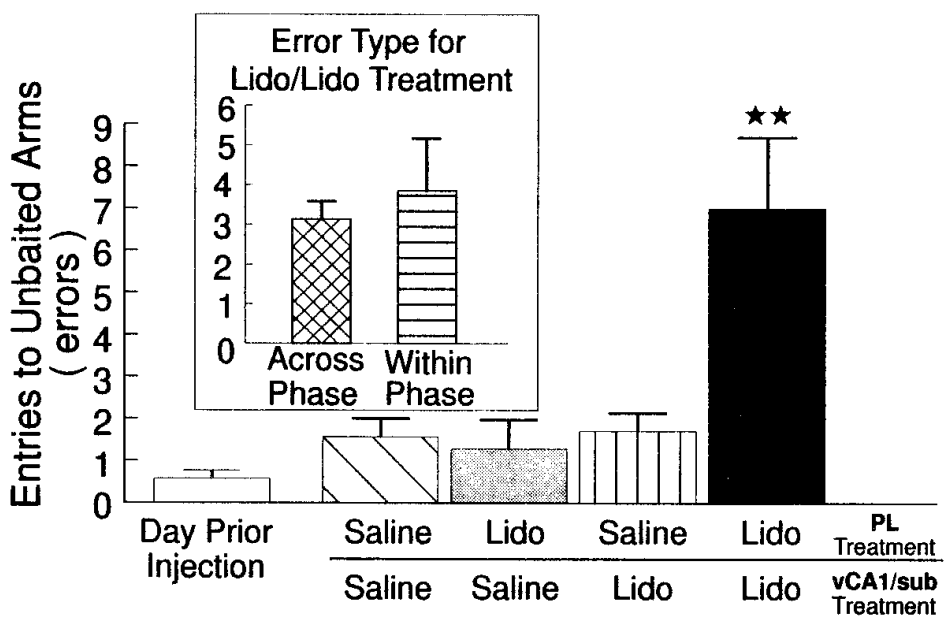

Figure 4. The effects of PL-vSub disconnections on performance of a radial-arm maze test battery. $A$, Nondelayed random foraging. Number of errors (mean \pm SEM) made by rats on the day before the first injection (open bar), after unilateral infusions of saline into both the PL and the vSub (hatched bar), unilateral infusions of lidocaine (Lido) into the PL and contralateral saline in the vSub (gray bar), unilateral infusions of Lido into the vSub and contralateral saline into the PL (stripped bar), and unilateral Lido into the vSub and contralateral Lido into the PL (disconnection; black bar) before the nondelayed RF task. $B$, Delayed spatial win-shift. Number of errors (mean \pm SEM) made by rats on the day before the first injection (open bar), after unilateral infusions of saline into both the PL and the vSub (hatched bar), unilateral infusions of lidocaine (Lido) into the PL and contralateral saline in the vSub (gray bar), unilateral infusions of Lido into the vSub and contralateral saline into the PL (stripped bar), and unilateral Lido into the vSub and contralateral Lido into the PL (disconnection; black bar) before the test phase of the delayed SWSh task. ${ }^{* *} p<0.001$ versus all other treatment conditions. Inset shows number of across-phase (cross-hatched bar) versus within-phase (horizontal-stripped bar) errors made by rats during Lido/Lido (disconnection) injection days. $C$, Location of cannulae tips (black circles) for all rats used for data analysis receiving PL-vSub disconnections before either the nondelayed RF task or the delayed SWSh task. Plates are computer-generated adaptations from Swanson (1992) that were modified to resemble those from Paxinos and Watson (1986). Numbers beside each plate correspond to millimeters from bregma. For clarity, $C$ represents the location of cannulae tips on sides that received infusions on disconnection injection days. All animals received infusions of either lidocaine or saline in each hemisphere.

not significant). A separate analysis confirmed that there were no significant differences between treatment conditions in the latency to reach the first food cup or on the average time per subsequent choice (all $F<1.1$, not significant).

Delayed SWSh. A separate group of rats $(n=7)$ with two sets of bilateral cannulae implanted into the PL, and the vSub received the injection protocol described above before the test phase of the delayed SWSh task on four occasions. The analysis revealed a highly significant main effect of Treatment $\left(F_{(4,12)}=10.99, p<\right.$ $0.001)$. Tukey's post hoc analysis for repeated measures revealed that rats made significantly more errors on the injection days in which the PL and the vSub were disconnected versus all other injection days ( $p<0.001$; see Fig. $4 B$ ). There were no other significant differences in the number of errors made on any of the other injection days. Subsequent planned comparisons on the type of errors made after PL-vSub disconnections showed no significant differences in the number of across- versus within-phase errors $\left(F_{(1,6)}=0.517\right.$, not significant; see Fig. $4 B$, inset $)$. Furthermore, there were no significant effects of Injection Order, Error type, or any significant interactions (all $F<2.1$, not significant). A separate series of tests was conducted to assess any hemispheric biases on the number of errors made after unilateral vSub inactivations, unilateral PL inactivations, and disconnection lesions. This analysis revealed no significant effects of the side of injection 
on the number of errors made by rats on the three injection days (all $F<1.0$, not significant).

Analysis of the latency data revealed no significant differences in the latency to reach the first food cup across all injection days $\left(F_{(4,24)}=0.098\right.$, not significant). In contrast, analysis of the average time per subsequent arm choice did show a significant effect of Treatment $\left(F_{(4,24)}=3.76, p<0.05\right)$. Tukey's post hoc test for repeated measures revealed that animals took significantly longer $(p<0.05)$ on average for each choice $(M=38.1 \mathrm{sec})$ after saline injections into both the PL and the vSub versus latencies on unilateral PL inactivation injection days $(M=23.3 \mathrm{sec})$ or the day before the first injection $(M=17.3 \mathrm{sec})$. Given that animals receiving saline treatments in all other experiments of the present study did not differ in this latency measure when compared to the day before the first injection, or to latencies on lidocaine injection days, it appears that increased response latency after saline/saline treatments in the PL and the vSub is an anomalous finding.

Histology. The location of the cannulae tips for all animals receiving PL-vSub disconnections are represented in Figure $4 C$. Bilateral placements in the vSub were similar to those observed in Experiment 1. Similarly, bilateral placements in the PL were within the same region of the PFC as those observed by Seamans et al. (1995). Note that Figure $4 C$ represents the asymmetric disconnection lesions (see legend for details).

\section{N.Acc.-vSub disconnections}

Nondelayed RF. One group of rats $(n=8)$ with two sets of bilateral cannulae implanted into the N.Acc. and the vSub received the injection protocol described above four times before a daily trial of the nondelayed RF task. Analysis of these data revealed a highly significant main effect of Treatment $\left(F_{(4,16)}=\right.$ $10.56, p<0.001)$. Tukey's post hoc test for repeated measures revealed that rats made significantly more errors after disconnection lesions versus all other injection days $(p<0.001)$, and no other injection days differed significantly from each other (see Fig. $5 A$ ). Subsequent planned comparisons of the type of errors made after bilateral saline or disconnection lesions revealed that rats made an equal number of reentries into baited and nonbaited arms after either bilateral saline infusions or disconnection lesions $\left(F_{(1,7)}=0.44\right.$, not significant; see Fig. $5 A$, inset $)$. There were no main effects of Injection Order, Error type, or any significant interactions (all $F \leq 1.6$, not significant). Similarly, there were no hemispheric biases on the number of errors made after either unilateral vSub inactivations, unilateral N.Acc. inactivations or vSub/N.Acc. disconnections (all $F<3.0$, not significant). In addition, there were no significant differences among all treatment conditions on the latencies to reach the first food cup or on the average time per subsequent choice (all $F<2.2$, not significant).

Delayed SWSh. A separate group of rats $(n=9)$ with two sets of bilateral cannulae implanted into the N.Acc. and the vSub received the injection protocol described above on four occasions before the test phase of the delayed SWSh task. Analysis of these data revealed no significant main effect of Treatment $\left(F_{(4,20)}=\right.$ 0.82, not significant; see Fig. $5 B$ ). There was a significant main effect of Error type $\left(F_{(1,5)}=15.05, p<0.05\right)$, indicating that animals made significantly more across-phase errors than withinphase errors during all treatment conditions. There were no significant main effects of Injection order or any significant interactions (all $F<1.0$, not significant). Similarly, there were no significant differences among treatment conditions in the latencies to reach the first food cup or on the average time per subsequent choice (all $F<1.7$, not significant).
Histology. The location of the cannulae tips for all animals receiving N.Acc.-vSub disconnections is represented in Figure $5 C$. Bilateral placements in the vSub were similar to those observed in Experiment 1. Similarly, bilateral placements in the N.Acc. were within the same regions of the ventral striatum as those observed by Seamans and Phillips (1994). Note that Figure 5C represents the asymmetric disconnection lesions (see legend for details).

\section{DISCUSSION}

The present results reveal that the vSub region of the hippocampus and the PL region of the PFC are part of a neural circuit through which spatial information acquired before a delay is used subsequently to locate food on a radial-arm maze. In contrast, foraging in the absence of information obtained before a delay appears to depend on a direct interaction between the hippocampus and the N.Acc., and there does not appear to be a role for the PFC. As may be expected, bilateral reversible lesions in the vSub disrupt foraging with or without a delay, whereas unilateral inactivation has no effect.

A detailed consideration of the different foraging strategies used by rats in the delayed versus nondelayed foraging tasks may explain the differences in the effects of vSub/PL as compared to vSub/N.Acc. disconnections. On the surface, it may appear that the delayed SWSh and the nondelayed RF tasks only differ in terms of the duration of the memory requirement. However, a key distinction between these tasks is that rats can predict the location of food on the maze at the start of the test phase of the delayed task, whereas no previous information about the location of food is available before a daily nondelayed RF trial. Previous studies suggest that insertion of a delay while rats are searching for food on a radial-arm maze biases rats to forage prospectively (Cook et al., 1985), because they can use information acquired before the delay to predict the probable location of food on the maze (Cook et al., 1985) (S. Floresco and A. Phillips, unpublished observations). In this way, the rat approaches arms that are anticipated to contain food. Conversely, continuous foraging behavior in the absence of previous information about the location of food biases an animal to forage retrospectively (Roberts and Smythe, 1979; Cook et al., 1985) (S. Floresco and A. Phillips, unpublished observations). On the nondelayed RF task, rats must first make choices randomly until they gain knowledge about arms that do or do not contain food. As the trial continues, rats can actively avoid arms entered previously. Given these distinctions between prospective and retrospective coding on a radial maze, it may be conjectured that a hippocampal-PFC circuit subserves prospective response strategies, whereas a direct connection between the hippocampus and the N.Acc. is involved in retrospective foraging in the absence of previous information about the location of food.

\section{Interactions between the vSub and the PL region of prefrontal cortex in delayed foraging}

The logic underlying the use of disconnection lesions to identify components of a functional neural circuit is based on the assumption that information is transferred serially from one structure to an efferent region, on both sides of the brain in parallel. Furthermore, the design assumes that dysfunction will result from blockade of neural activity at the origin of a pathway in one hemisphere and the termination of the efferent pathway in the contralateral hemisphere. It follows that a unilateral inactivation at either site should have no effect on behavior. Using the present study as an example, if successful performance on a task is dependent on a serial connection linking the hippocampus to the PFC, then a 

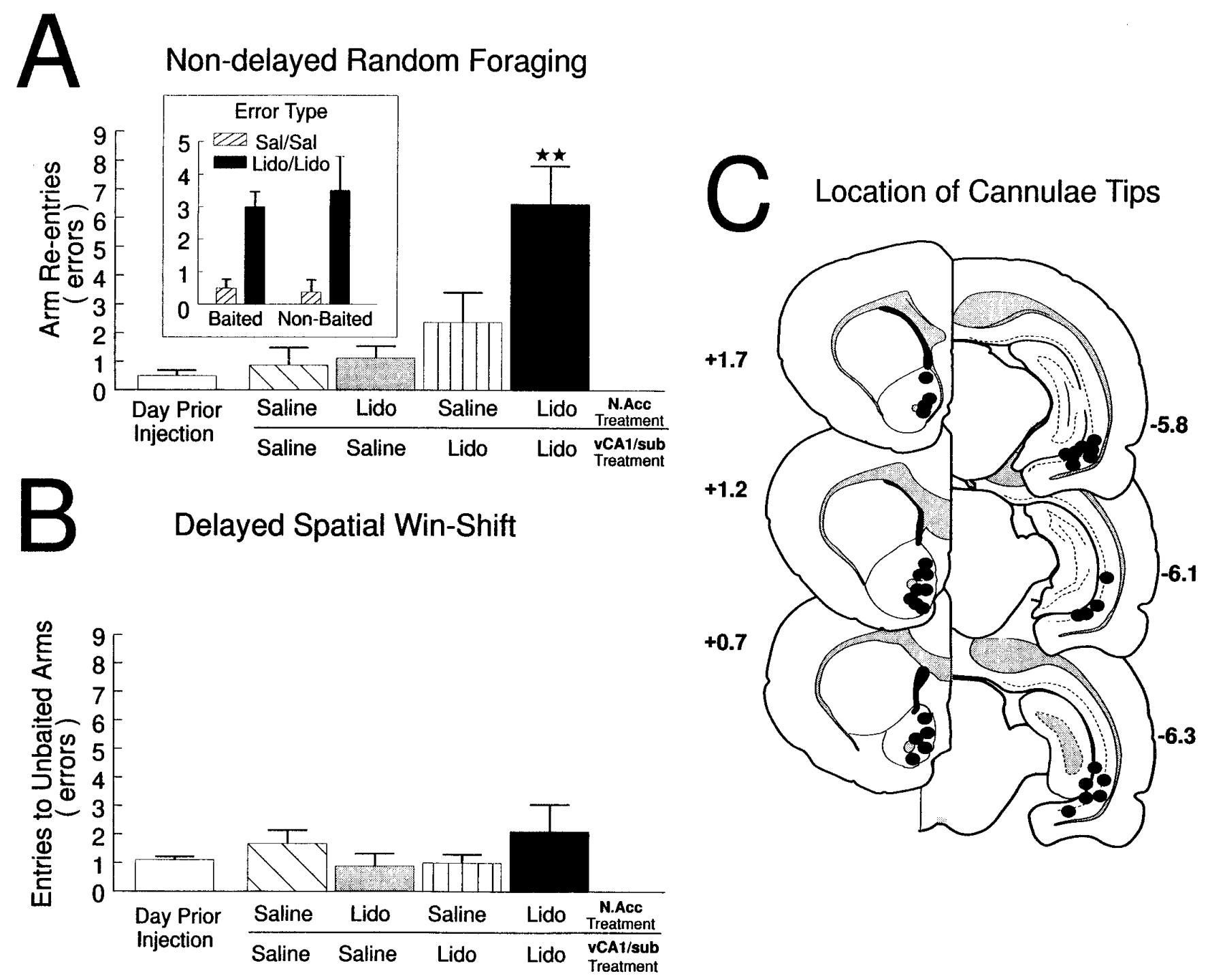

Figure 5. The effects of N.Acc.-vSub disconnections on performance of a radial-arm maze test battery. $A$, Nondelayed random foraging. Number of errors (mean \pm SEM) made by rats on the day before the first injection (open bar), after unilateral infusions of saline into both the N.Acc. and the vSub (hatched bar), unilateral infusions of lidocaine (Lido) into the N.Acc. and contralateral saline in the vSub ( gray bar), unilateral infusions of Lido into the vSub and contralateral saline into the N.Acc. (stripped bar), and unilateral Lido into the vSub and contralateral Lido into the N.Acc. (disconnection; black bar) before the nondelayed RF task. ${ }^{* *} p<0.001$ versus all other treatment conditions. Inset shows number of reentries to baited arms and nonbaited arms on saline/saline (hatched bar) and Lido/Lido disconnection (black bar) injection days. B, Delayed spatial win-shift. Number of errors (mean \pm SEM) made by rats on the day before the first injection (open bar), after unilateral infusions of saline into both the N.Acc. and the vSub (hatched bar), unilateral infusions of lidocaine (Lido) into the N.Acc. and contralateral saline in the vSub ( gray bar), unilateral infusions of Lido into the vSub and contralateral saline into the N.Acc. (stripped bar), and unilateral Lido into the vSub and contralateral Lido into the N.Acc. (disconnection; black bar) before the delayed SWSh task. $C$, Location of cannulae tips (black circles) for all rats used for data analysis receiving N.Acc.-vSub disconnections before either the nondelayed RF task or the delayed SWSh task. Plates are computer-generated adaptations from Swanson (1992) that were modified to resemble those from Paxinos and Watson (1986). Numbers beside each plate correspond to millimeters from bregma. For clarity, $C$ represents the location of cannulae tips on sides that received infusions on disconnection injection days. All animals received infusions of either lidocaine or saline in each hemisphere.

unilateral lesion of the vSub would prevent the PFC in the ipsilateral hemisphere from gaining access to information needed to solve the task. In the other hemisphere, information would be relayed from the intact vSub; however, it would go to a dysfunctional PFC. Thus, after this asymmetric disconnection, the PFC on both sides of the brain would be deprived of information essential to form an efficient foraging strategy. The use of different behavioral tasks permits further refinement in specifying the function of the neural circuit in question. In the present study, reversible disconnection lesions produced by lidocaine infusion into the unilateral vSub and contralateral PL produced a selective and significant disruption of foraging only during the test phase of the delayed SWSh task, indicating that afferents arising from the vSub and terminating in the PL are essential for performance of this task (Fig. 6B). In contrast, similar disconnections had no effect on foraging in the nondelayed RF task, in which the animal had no previous information about the location of food, suggesting that foraging behavior based on a "retrospective" strategy is not dependent on information from the hippocampus reaching the PL (Fig. 6C).

Reversible disconnection lesions between the vSub and the PL produced precisely the same pattern of results as those 


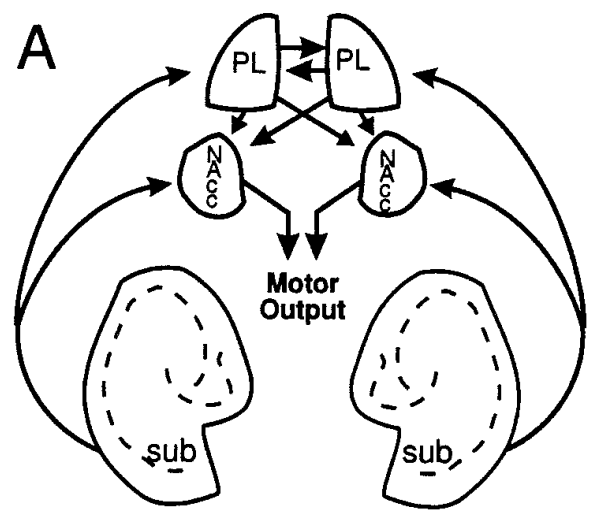

Figure 6. Diagram of the anatomical connections investigated in the present study between the vSub, the PL, and the N.Acc. $\times$ represents the location of the unilateral inactivations to the vSub and PL or N.Acc. for each task. Solid arrows represent intact pathways. Open arrows represent pathways that are not blocked but do not carry the relevant spatial information because of a concomitant lidocaine-induced lesion upstream of this pathway. T-symbols represent blocked, nonfunctional pathways. $A$, An overview of the ipsi- and contralateral connections between the three brain regions. Note the unilateral projections from vSub to the forebrain and the contralateral projections between the PL and its connections to the N.Acc. B, Proposed route of information transfer between the vSub and PL during the delayed SWSh task. By disconnecting the PL-vSub pathway, information cannot be processed by the PL to generate appropriate responses after a delay, thereby disrupting appropriate output (impairment). $C$, Proposed route of information transfer between the vSub and the PL during the nondelayed RF task. By disconnecting the PL-vSub pathway, information is still able to access the N.Acc., thereby allowing for appropriate output (no impairment). $D$, Proposed route of information transfer between the vSub and N.Acc. during the nondelayed RF task. Disconnection of the N.Acc.-vSub pathway prevents the flow of information from the vSub through the N.Acc. to motor output centers (impairment). E, Proposed route of information transfer between the vSub and N.Acc. during the delayed SWSh task. Information from the vSub may be routed primarily through the PL and subsequently to the N.Acc. Thus, even though the pathway from the vSub to the N.Acc. is disconnected, spatial information may still be transferred from the unanesthetized vSub to the ipsilateral PL and subsequently routed to the contralateral N.Acc., allowing for appropriate output (no impairment).

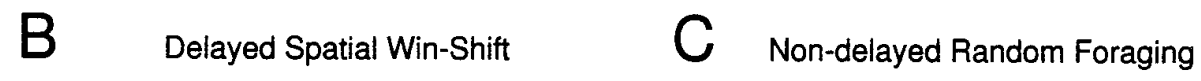

$\mathrm{PL}$ - vCA1/sub Disconnections
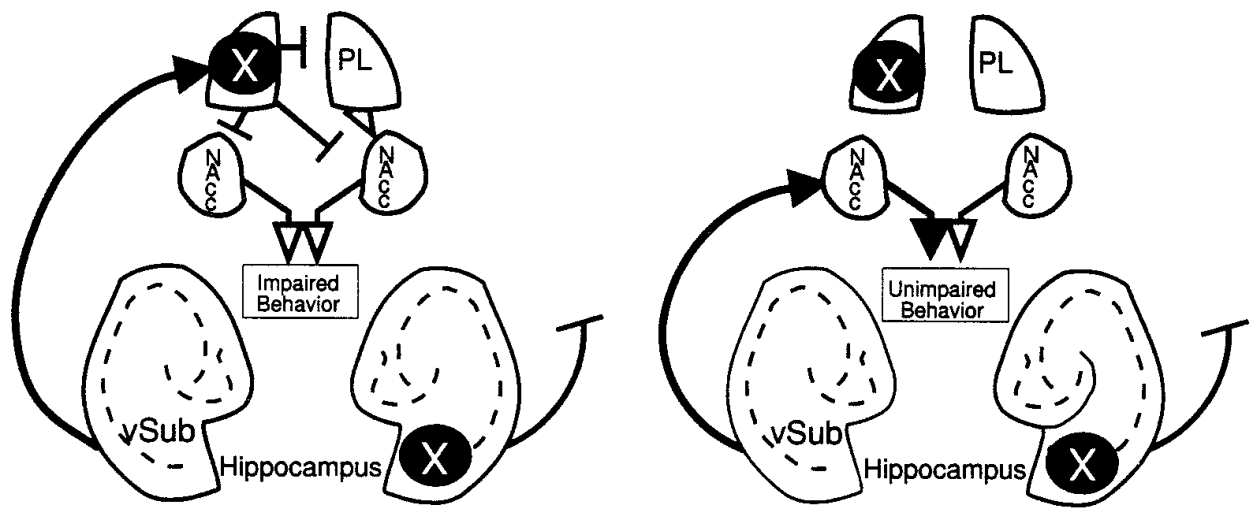

D Non-delayed Random Foraging E Delayed Spatial Win-Shift N.Acc - vCA1/sub Disconnections

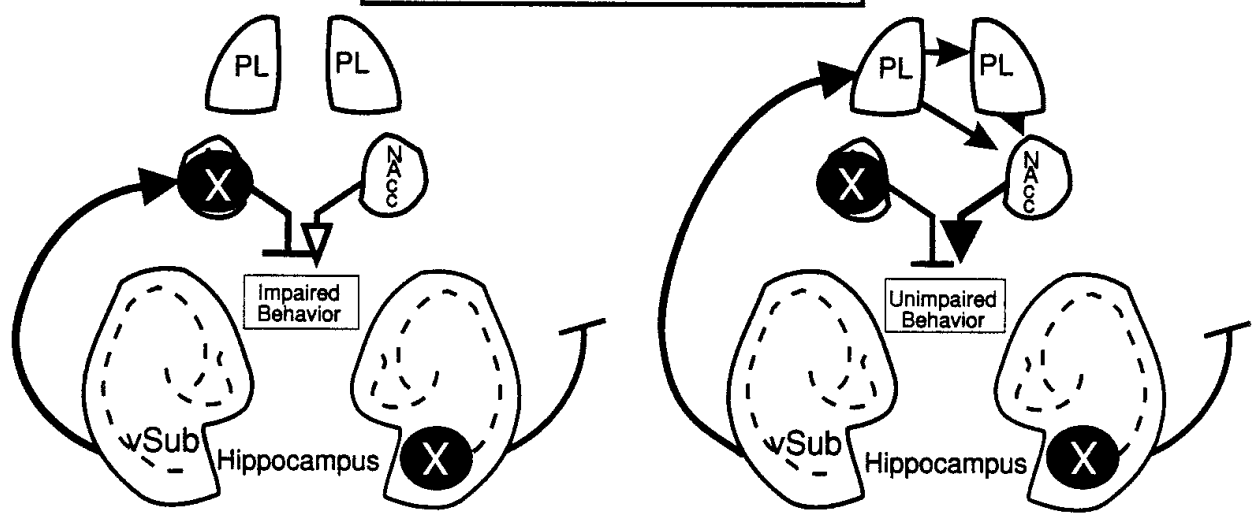

observed after bilateral lidocaine infusions into the PL region of the rat PFC (Seamans et al., 1995). On the basis of those results, it was suggested that the PL plays a role in the retrieval of spatial information required to predict the probable location of food during the test phase of the delayed SWSh task. Furthermore, it was conjectured that spatial information, stored in the temporal lobe during the $30 \mathrm{~min}$ delay, may be accessed by the PL, which organizes the prospective sequence of motor responses necessary for efficient foraging behavior.
The present results with the vSub-PL disconnection lesions further substantiate this hypothesis. As such, these data also provide direct support for theories suggesting that the neural circuit linking the hippocampus and PFC provides an essential pathway by which spatial information can be integrated into the cognitive and motor planning processes mediated by the PFC (Goldman-Rakic, 1987; Fuster, 1991; Doyère et al., 1993). These findings are also consistent with other theories implicating the rat PFC by itself, or its interactions with the hippocam- 
pus, in the mediation of planning (Kesner and DiMattia, 1987; Granon and Poucet, 1995).

One point that deserves further consideration is the finding that rats made a significant number of within-phase errors after PL/vSub disconnections on the delayed task (Fig. $5 B$, inset), whereas similar lesions did not cause a significant increase in reentry errors on the nondelayed task (Fig. 5A). It must be noted that a comparable number of across-phase errors was also recorded on the delayed tasks. This pattern of results was also observed after bilateral inactivations of the PL (Seamans et al., 1995) and highlights the differences between the delayed and nondelayed tasks. It is important to emphasize that a comparable number of within- and across-phase errors on the delayed task, coupled with few reentry errors on the nondelayed task, is indicative of a failure to execute a prospective foraging strategy, not an inability to remember recent arm choices during a trial. Therefore, these data again are consistent with the hypothesis that circuits linking the PL and vSub are involved in the execution of a prospective foraging strategy rather than the retention of information over a delay. Inactivations of the PL bilaterally, or disconnections of the vSub and PL, may have caused both across- and withinphase errors on the delayed task because they severely disrupted the execution of the prospective foraging strategy, which is required to solve the task.

The present study differed from most other delayed-response tasks that have been used to assess the role of the PFC in short-term memory processes in the use of a longer, 30 min delay interval, compared to $<60 \mathrm{sec}$ used in previous studies. Electrophysiological studies have shown that information relating to the spatial location of a stimulus over a short delay is encoded in the electrical activity of subsets of neurons in the dorsolateral PFC (Goldman-Rakic, 1990). This activity is thought to provide a neural substrate for the shortterm retention of information required for the generation of a subsequent motor response and can be disrupted by reversible inactivations of the temporal cortex (Fuster et al., 1985). Previous data indicate that spatial information, such as that obtained in the delayed SWSh task, is not stored in the PFC but, rather, is accessed by the $\mathrm{PFC}$ at a time when prospective planning is dependent on recently acquired spatial information (Seamans et al., 1995). This dependence on a cortical network that spans several cortical areas is consistent with Fuster's (1995) conceptual framework of a distributed memory system in which memories are "content-addressed" by many associated elements and may be activated by one aspect of this configuration (i.e., spatial location). Once activated, the specific memory can be maintained by neural activity in local and transcortical reentrant circuits. The present data support the idea that reciprocal connections between the vSub-PFC-temporal lobe via multisynaptic connections through the entorhinal cortex (Amaral and Witter, 1994) could provide a distributed neural network involving the frontal and temporal cortices, underlying the retrieval and use of trial-unique spatial information over extended delays.

\section{A role for the hippocampal-ventral striatal pathway in spatially mediated foraging behavior}

Disconnections between the vSub and the N.Acc. disrupted foraging behavior in rats during the nondelayed RF task. These data support the hypothesis that inputs from the vSub to the N.Acc. are involved in the initiation and guidance of exploratory locomotion (Mogenson et al., 1993). Chemical stimulation of the vSub results in an increase in exploratory behavior in an open field, which is attenuated after pharmacological manipulations within the N.Acc. (Yang and Mogenson, 1987). The present data expand on Mogenson's initial hypothesis by suggesting that the hippocampal-N.Acc. pathway also is involved in goal-directed food searching behavior in a complex, spatially cued environment when an animal initially has no previous information about the location of food. This conjecture regarding hippocampal-ventral striatal interactions is supported further by the observation that N.Acc. neurons recorded in rats foraging on a radial-arm maze demonstrate place- and movement-correlated activity (Lavoie and Mizumori, 1994), as do cells in the vSub (Barnes et al., 1990; Jung et al., 1994; Poucet et al., 1994). Moreover, the fact that disconnections between the PL and the vSub did not disrupt foraging behavior on the nondelayed RF task strongly suggests that, when rats do not have previous information about the location of food, the direct transfer of spatial information from the vSub to the N.Acc. is used to control efficient foraging behavior. Accordingly, a unilateral inactivation of the vSub could deprive the N.Acc. in one hemisphere of essential spatial information, whereas direct inactivation of the contralateral N.Acc. blocks the integration of this information into accurate goal-directed responses, thereby leading to random responding and impaired foraging behavior (Fig. 6D).

Disconnections between the N.Acc. and the vSub before the test phase of the delayed SWSh did not disrupt foraging behavior, suggesting that direct inputs from the hippocampus to the N.Acc. are not essential for efficient performance on this delayed response task. This result may seem paradoxical, considering that bilateral inactivation of either the vSub (present study) or the N.Acc. (Seamans and Phillips, 1994) severely disrupted test phase performance on this task. However, given that an intact PL is also crucial for efficient delayed SWSh behavior (Seamans et al., 1995), it appears that efferents from the PL to the N.Acc. play a critical role when foraging is guided by previous knowledge of the probable location of food in the test environment. Moreover, the present data demonstrate that inputs from the vSub to the PL are also essential for delayed SWSh performance. Given these results, it can be inferred that after a vSub-N.Acc disconnection, unimpaired transmission of information between the intact hippocampus and the PFC would still occur, thereby enabling the relevant spatial information to be transferred serially first from the vSub to the PL and then to the unanesthetized N.Acc. via either ipsilateral or contralateral cortico-striatal connections (Sesack et al., 1989; Brog et al., 1993; Conde et al., 1995) [see Fig. $6 E$ (also $6 A)$ ].

\section{Conclusions}

Goldman-Rakic has posited that "the hippocampus and PFC are functionally as well as anatomically related and, in general, that the PFC regulates behavior in collaboration with a large set of other cortical and subcortical structures, which together constitute the brain's machinery for spatial cognition" (Goldman-Rakic, 1994, p. 352). The present series of experiments provides direct confirmation of this hypothesis by demonstrating that performance of a delayed spatial task that requires an animal to use previously acquired trial-unique information is dependent on a network comprising the vSub and the PL. Furthermore, our data expand on this idea by showing that exploratory goal-directed locomotion that is not dependent on previously acquired knowledge about the location of food is subserved by a separate subcortical network linking of the hippocampus to the ventral striatum. Finally, with respect to delayed spatial tasks, these results suggest that interactions between the PFC and the ventral striatum are involved in the transformation of spatial memory, processed by hippocampal-cortical circuits, into an efficient sequence of goal-directed motor responses (Robbins, 1990, 1991; GoldmanRakic et al., 1992). Further investigation of this hypothesis is the subject of ongoing research in our laboratory. 


\section{REFERENCES}

Albert DJ, Madryga FJ (1980) An examination of the functional spread of $4 \mu \mathrm{l}$ of slowly infused lidocaine. Behav Neural Biol 29:378-384.

Amaral DG, Witter MP (1994) Hippocampal formation. In: The rat nervous system, 2nd Ed (Paxinos G, ed), pp 443-494. San Diego, Academic.

Barnes CA, McNaughton BL, Mizumori SJ, Leonard BW, Lin LH (1990) Comparison of spatial and temporal characteristics of neuronal activity in sequential stages of hippocampal processing. Prog Brain Res 83:287-300.

Brog JS, Salyapongse A, Deutch A, Zahm DS (1993) The pattern of afferent innervation of the core and shell in the "accumbens" part of the ventral striatum: immunohistochemical detection of retrogradely transported fluoro-gold. J Comp Neurol 338:255-278.

Conde F, Maire-Lepoivre E, Audinat E, Crepel F (1995) Afferent connections of the medial frontal cortex of the rat. II. Cortical and subcortical afferents. J Comp Neurol 325:567-593.

Cook RG, Brown RF, Riley DA (1985) Flexible memory processing by rats: use of prospective and retrospective information in the radial arm maze. Anim Behav Proc 11:453-469.

Devenport LD, Devenport JA, Halloway FA (1981) Reward-induced stereotypy: modulation by the hippocampus. Science 212:1288-1289.

Doyère V, Burette F, Negro CR, Laroche S (1993) Long-term potentiation of hippocampal afferents and efferents to prefrontal cortex: implications for associative learning. Neuropsychologia 31:1031-1053.

Dunnett SB (1990) Role of the prefrontal cortex and striatal output systems in short-term memory deficits associated with aging, basal forebrain lesions, and cholinergic-rich grafts. Can J Psychol 44:210-232.

Everitt BJ, Morris KA, O'Brien A, Burns L, Robbins TW (1991) The basolateral amygdala-ventral striatal systems and conditioned place preference: further evidence of limbic-striatal interactions underlying reward-related processes. Neuroscience 41:1-18.

Floresco SB, Seamans JK, Phillips AG (1996) Differential effects of lidocaine infusions into the ventral CA1/subiculum or the nucleus accumbens on the acquisition and retention of spatial information. Behav Brain Res 81:163-172.

Fuster JM (1991) The prefrontal cortex and its relation to behavior. Prog Brain Res 87:201-211.

Fuster JM (1995) Memory and planning: two temporal perspectives of frontal lobe function. Adv Neurol 66:9-19.

Fuster JM, Bauer RH, Jervey JP (1985) Functional interactions between inferotemporal and prefrontal cortex in a cognitive task. Brain Res 330:299-307.

Gaffan D, Harrison S (1987) Amygdalectomy and disconnection in visual learning for auditory secondary reinforcement by monkeys. J Neurosci 7:2285-2292.

Gaffan D, Gaffan EA, Harrison S (1988) Disconnection of the amygdala from visual association cortex impairs visual reward-association learning in monkeys. J Neurosci 8:3144-3150.

Gaffan D, Gaffan EA, Harrison S (1989) Visual-visual associative learning and reward-association learning in monkeys: the role of the amygdala. J Neurosci 9:558-564.

Goldman-Rakic PS (1987) Circuitry of the prefrontal cortex and its regulation of behavior by representational knowledge. In: Handbook of physiology, Vol 5 (Mountcastle PF, ed), pp 373-417. Bethesda, MD: American Physiological Association.

Goldman-Rakic PS (1990) Cellular and circuit basis of working memory in prefrontal cortex of nonhuman primates. Prog Brain Res 85:325-335.

Goldman-Rakic PS (1994) Working memory dysfuntion in schizophrenia. J Neuropsychol Clin Neurosci 6:348-357.

Goldman-Rakic PS, Bates JF, Chafee MW (1992) The prefrontal cortex and internally generated motor acts. Curr Opin Neurobiol 2:803-835.

Granon S, Poucet B (1995) Medial prefrontal lesions in the rat and spatial navigation: evidence for impaired planning. Behav Neurosci 109:474-484.

Groenewegen HJ, Vermeulen-Van der Zee E, Te Kortschot A, Witter MP (1987) Organization of the projections from the subiculum to the ventral striatum in the rat: a study using anterograde transport of Phaseolus vulgarus leucoagglutinin. Neuroscience 23:103-120.

Isaacson RL (1982) The hippocampus. In: The limbic system, 2nd Ed. New York: Plenum.

Jarrard LE (1993) On the role of the hippocampus in learning and memory in the rat. Behav Neural Biol 60:9-26.

Jay TM, Witter MP (1991) Distribution of hippocampal CA1 and subic- ular efferents in the prefrontal cortex of the rat studied by means of anterograde transport of Phaseolus vulgaris leucoagglutinin. J Comp Neurol 313:574-586.

Jung MW, Wiener SI, McNaughton BL (1994) Comparison of spatial firing characteristics of units in dorsal and ventral hippocampus of the rat. J Neurosci 14:7347-7356.

Kelley AE, Stinus L (1985) Disappearance of hoarding behavior after 6-hydroxydopamine lesions of the mesolimbic dopamine neurons and its reinstatement with L-dopa. Behav Neurosci 99:531-545.

Kesner RP (1989) Retrospective and prospective coding of information: role of the medial prefrontal cortex. Exp Brain Res 74:163-167.

Kesner RP, DiMattia BV (1987) Neurobiology of an attribute model of memory. In: Progress in psychobiology and physiological psychology (Morrison AR, Epstein AN, eds), pp 207-277. New York: Academic.

Lavoie AM, Mizumori SJ (1994) Spatial movement- and reward-sensitive discharge by medial ventral striatum neurons in rats. Brain Res 638:157-68.

Mogenson GJ, Brudzynski SM, Wu M, Yang CR, Yim CY (1993) From motivation to action: a review of dopaminergic regulation of limbic $\rightarrow$ nucleus accumbens $\rightarrow$ ventral pallidum $\rightarrow$ pedunculopontine nucleus circuitries involved with limbic-motor integration. In: Limbicmotor circuits and neuropsychiatry (Kalivas PW, Barnes CD, eds), pp 193-263. Boca Raton, FL: CRC.

Morris RGM, Schenk F, Tweedie F, Jarrard LE (1991) Ibotenate lesions of the hippocampus and/or subiculum: dissociating components of allocentric spatial learning. Eur J Neurosci 2:1016-1028.

O'Keefe J, Nadel L (1978) The hippocampus as a cognitive map. Oxford: Oxford UP.

Olton DS, Papas BC (1979) Spatial memory and hippocampal function. Neuropsychologia 17:669-682.

Olton DS, Samuelson RJ (1976) Remembrance of places past: spatial memory in rats. Anim Behav Proc 2:97-116.

Packard MG, Hirsh R, White NM (1989) Differential effects of fornix and caudate nucleus lesions on two radial arm maze tasks: evidence for multiple memory systems. J Neurosci 9:465-1472.

Packard MG, Regenold W, Quirion R, White NM (1990) Post-training injection of the acetylcholine M2 receptor antagonist AF-DX 116 improves memory. Brain Res 524:72-76.

Paxinos G, Watson C (1986) The rat brain in stereotaxic coordinates, 2nd Ed. New York: Academic.

Poucet B, Herrmann T, Buhot MC (1991) Effects of short lasting inactivations of the ventral hippocampus and medial septum on long term and short term acquisition of spatial information in rats. Behav Brain Res 4:453-65.

Poucet B, Thinus-Blanc C, Muller RU (1994) Place cells in the ventral hippocampus of rats. NeuroReport 5:2045-2048.

Robbins TW (1990) The case for frontalstriatal dysfunction in schizophrenia. Schizophrenia Bull 16:391-402.

Robbins TW (1991) Cognitive deficits in schizophrenia and Parkinson's disease: neural basis and the role of dopamine. In: The mesolimbic dopamine system: from motivation to action (Willner P, Scheel-Kruger J, eds), pp 497-528. New York: Wiley.

Roberts WA, Smythe WE (1979) Memory for list of spatial events in the rat. Learn Motiv 8:341-351.

Seamans JK, Phillips AG (1994) Selective memory impairments produced by transient lidocaine-induced lesions of the nucleus accumbens in rats. Behav Neurosci 108:456-468.

Seamans JK, Floresco SB, Phillips AG (1995) Functional differences between the prelimbic and anterior cingulate regions of rat prefrontal cortex. Behav Neurosci 109:1063-1073.

Sharp PE, Green C (1994) Spatial correlates of firing patterns of single cells in the subiculum of freely moving animals. J Neurosci $14: 2239-2356$.

Sesack SR, Deutch AY, Roth RH, Bunney BS (1989) Topographical organization of the efferent projections of the medial prefrontal cortex in the rat: an anterograde tract-tracing study with Phaseolus vulgaris leucoagglutinin. J Comp Neurol 290:213-242.

Swanson LW (1992) Structure of the rat brain. Amsterdam: Elsevier.

Welsh JP, Harvey JA (1991) Pavlovian conditioning in the rabbit during inactivation of the interpositus nucleus. J Physiol (Lond) 444:459-480.

Yang CR, Mogenson GJ (1987) Hippocampal signal transmission to the mesencephalic locomotor regions and its regulation by dopamine D-2 receptors in the nucleus accumbens: an electrophysiological and behavioral study. Neuroscience 23:1041-1055. 